\title{
BREATHE OUT: A Randomized Controlled Trial of a Structured Intervention to Improve Clinician Satisfaction With "Difficult" Visits
}

\author{
Jennifer Y. C. Edgoose MD, MPH, Caitlin J. Regner, BS, and Larissa I. Zakletskaia, MA
}

Purpose: Difficult patients comprise at least $\mathbf{1 5 \%}$ of ambulatory visits and are associated with clinician burnout. No structured procedure has been reported to assist health care practitioners with these challenging relationships. This randomized trial evaluated whether a pre- and postvisit patient-centered and clinician-reflective technique called BREATHE OUT improved clinician satisfaction during visits with patients perceived by the clinician as difficult.

Methods: Six family medicine residency clinics paired as urban, suburban, and rural sites were randomized by clinic to the BREATHE OUT intervention or usual care of difficult patients. A total of 57 physician faculty, nurse practitioners, physician assistants, and residents participated. The validated Physician Satisfaction Scale (PSS) was administered and analyzed using hierarchical linear modeling to assess differences between the intervention and control groups.

Results: The BREATHE OUT intervention improved the PSS score $(P=.02)$ in the intervention group compared with the usual care group. Female practitioners reported worse PSS scores compared with their male counterparts despite whether they used BREATHE OUT $(P=.009)$.

Conclusions: Application of the BREATHE OUT questionnaire before and after visits improved clinician satisfaction with difficult patient encounters. (J Am Board Fam Med 2015;28:13-20.)

Keywords: Burnout, Professional; Communication; Doctor-Patient Relations; Medical Education; Physicians, Family

Any practicing clinician can easily recall a personal experience with a so-called "difficult" patient. Difficult patients make up at least 1 of every 6 ambulatory visits. ${ }^{1,2}$ While poetically described as "heartsink" patients a quarter century ago in the British Medical fournal, ${ }^{3}$ these patients are even recognizable in accounts from the fifth century BC, when Sophocles depicted Philoctetes as a demanding character in chronic pain from a festering wound. ${ }^{4}$ Prior studies established characteristics as-

This article was externally peer reviewed.

Submitted 14 December 2013; revised 15 July 2014; accepted 22 July 2014.

From the Department of Family Medicine, University of Wisconsin-Madison (JYCE, LIZ); and the University of Wisconsin School of Medicine and Public Health, Madison (CJR).

Funding: This project was supported by the University of Wisconsin Department of Family Medicine Scholarly Small Grant Program.

Conflict of interest: none declared.

Corresponding author: Jennifer Y. C. Edgoose, MD, MPH, Department of Family Medicine, University of Wisconsin, 1100 Delaplaine Ct, Madison, WI 53715-1896 (E-mail: jennifer.edgoose@fammed.wisc.edu). sociated with difficult patients, including mental health disorders, multiple symptoms, chronic pain, poor functional status, unmet expectations, and high utilization of health care services. ${ }^{1,2,5}$ On the other hand, clinicians were more likely to consider their patients as difficult if they were younger, had lower job satisfaction and poorer psychosocial attitudes, and were earlier in their medical careers. ${ }^{2,6,7}$ Physicians who believe they have a large number of difficult patients are 12 times more likely to experience burnout. ${ }^{8}$

In the 1950s Michael Balint ${ }^{9,10}$ was the first to study difficult patients and their relationships with their general practitioners through the lens of psychoanalysis. Elder et al ${ }^{11}$ more recently interviewed 102 highly respected family physicians with at least 10 years of experience from around the United States and found that difficult patient encounters were associated with opposition, misuse of power, and compassion fatigue, whereas success in working with difficult patients involved collaboration, appropriate use of power, and empathy. In tribute 
to Balint's original work, physicians form "Balint groups" to address a variety of medical concerns, including difficult patients. ${ }^{11}$ While these small groups have explored issues around the doctorpatient relationship, there currently exists no formal procedure to assist clinicians in achieving the successful strategies outlined by Elder et al. ${ }^{12}$

To address this knowledge gap, the principal investigator (PI) (JYCE) developed a structured intervention called BREATHE OUT (see Appendix), which prompts active reflection on bias, agenda, and context; gathering of a patient's social history; mindful breathing; and an openness to surprise. A feasibility before-and-after cohort pilot study was conducted in 2010 to test the BREATHE OUT intervention among 23 eligible clinicians, including faculty, residents, and advanced practitioners, at a family medicine residency clinic. The Physician Satisfaction Scale (PSS) ${ }^{13}$ was used to assess the tool (further described in Methods). Completion of the BREATHE OUT intervention proved to be both efficient, requiring $<3$ minutes, and effective in improving clinicians' satisfaction with their difficult patients (PSS score of $37 \pm 6$ in the intervention group [ $\mathrm{n}=18]$ and $48 \pm 5$ in the control group [n $=15[; P<.001$ ); a lower PSS score indicates more positive/favorable outcomes. Based on these promising results, we tested the hypothesis that the BREATHE OUT structured intervention will improve clinician satisfaction with clinician-identified difficult patients compared with clinicians not using any intervention.

\section{Methods}

This is a randomized, prospective, controlled trial studying the effect of the use of the BREATHE OUT intervention on clinician satisfaction with their difficult patients. This study was reviewed and approved by the University of Wisconsin-Madison health sciences human subjects institutional review board.

\section{Subjects and Setting}

One of the strongest associations with difficult patients is provider inexperience ${ }^{7}$; therefore we chose to conduct our study in residency clinics that offer inexperienced learner clinicians as well as seasoned faculty. Six family medicine residency clinics from the PI's statewide department participated; provider subjects ranged from first-year residents to physicians who had been in practice $>15$ years. The study was conducted over a 5 -week period from November to December 2012, and the 6 clinics were selected and paired based on urban, suburban, or rural settings. A senior researcher not involved in data collection or analysis tossed a coin to assign clinics in each pair to the intervention or control group; assignments were revealed to the clinics after they accepted the invitation to participate in the study.

\section{BREATHE OUT: Development and Description}

BREATHE OUT was developed by the PI based on aspects of both clinician self-reflection and patient-centeredness. A central principal of Balint's ${ }^{9,10}$ original work is clinician self-awareness within the clinician-patient dyad. In his seminal article on mindful practice Epstein ${ }^{14}$ similarly wrote "perhaps lack of self-awareness is why physicians more often espouse these values [of empathy, compassion, and altruism] than demonstrate them and why they tend to be less patient-centered and confuse their own perspectives with those of the patient in situations that involve conflict and strong emotions." Likewise, Elder et $\mathrm{al}^{11}$ found that those physicians known to work successfully with difficult patients "mentioned 'psyching up' by checking their own attitudes and recognizing their own biases, as well as trying to separate their own emotions from those of the patient, remaining open to surprises, and even using breathing exercises." Acknowledging these important practices, the BREATHE OUT intervention includes reflection of the clinician's own biases and agenda(s) and instructs them to take a moment to pause and literally breathe before entering the examination room.

In these challenging doctor-patient relationships, attending to the patient is no less important. Evidence shows strong patient-centered relationships promote greater satisfaction for patients and providers. ${ }^{15}$ Application of the Physician Belief Scale, a measure of clinician psychosocial orientation, demonstrated that a greater psychosocial orientation correlated with a more patient-centered communication style, ${ }^{16,17}$ whereas physicians with poorer scores on the Physician Belief Scale had more difficult patient encounters. ${ }^{7}$ Thus the BREATHE OUT intervention is also designed to promote patient-centeredness. This includes gathering a psychosocial history to explore context, intent, and meaning of the presenting concern; 
articulating the patient's agenda as distinct from the clinician's; and suggesting that the clinician be open to the unexpected.

The BREATHE OUT structured intervention incorporates the aforementioned strategies through 2 brief sets of questions (Appendix) intended for completion by practitioners before and after a clinical encounter with a difficult patient. BREATHE is a mnemonic for a previsit procedure to be completed by the clinician upon identifying a difficult patient on his or her schedule. The previsit components are to (1) list at least $1 \mathrm{Bias} /$ assumption you have about this patient; (2) REflect on why you identify this patient as "difficult"; (3) list 1 thing you'd like to Accomplish today; (4) THink about 1 question you'd like to address today that would enable you to further explore your assumptions; and (5) stop before you Enter the examination room and take 3 deep breaths, in through your nose and out through your mouth.

OUT is a mnemonic for a postvisit procedure to be completed as the clinician goes "out" of the clinic examination room. The postvisit components are to (1) reflect on the Outcome of the encounter (considering the patient's and your own agenda); (2) did you learn anything Unexpected?; and (3) list 1 thing you look forward to addressing if you were to run into this patient Tomorrow.

\section{Outcome Measures}

\section{Physician Satisfaction Scale}

To quantify clinician satisfaction after the difficult patient encounter, we used the PSS, ${ }^{13}$ a validated, 16-item Likert-scale survey consisting of $10 \mathrm{pa}-$ tient-related items and 6 contextual items with an internal reliability (Cronbach $\alpha$ ) of 0.85 . As an example, the PSS evaluates the physician's emotional state ("Emotionally, I was very comfortable during this visit"); confidence ("This visit made me feel good about being a doctor"); and feelings about the previous patient encounter ("My previous patient encounter went very well"). Subjects rated how strongly they agreed or disagreed with the PSS items on a scale of 1 to 5 . Responses to the negatively phrased PSS items were reverse coded for analysis, and all responses to the16 PSS items were summed up to produce a summary score. There are 80 maximum possible points in the PSS scale; 16 is the minimum possible score. The PSS scale was validated as a continuous response measure. A lower PSS score indicates a more positive/favorable outcome.

\section{Individual Characteristics}

A brief demographics survey was attached to each PSS, which collected participants' sex; job title (resident, fellow, attending physician, advanced practitioner); and experience level (resident physicians indicated their postgraduate year and faculty attending physicians provided how many years they were after their residency).

\section{Study Procedures}

Clinic staff, including all providers, received an E-mail explaining the study's purpose, background, protocol, and risks and benefits. A standardized invitational presentation was also given by the PI and research assistant at each clinic site; this reiterated the E-mail and included the general study protocol for both the control and intervention groups without actually showing the BREATHE OUT mnemonic. Each clinic was given a sealed envelope at the end of the talk, which revealed whether they were the intervention or control group. Intervention groups then were introduced to the BREATHE OUT questionnaire and given the opportunity to ask questions regarding its use, but no formal training other than the study protocol was provided.

Participants were eligible for inclusion if they were a practicing clinician seeing patients during the study period at one of the participating clinics, including faculty physicians, advanced registered nurse practitioners, physician assistants, and family medicine residents and fellows. Each participant was assigned a unique personal identifier consisting of the first 2 letters of the mother's maiden name and the last 2 numbers of their phone number. This identifier allowed us to account for clinicians who completed the PSS multiple times.

The study protocol is shown in Figure 1. At both intervention and control sites, clinicians reviewed their half-day (morning or afternoon) schedules during a routine huddle with their nurse or medical assistant. Patients were identified as difficult by the clinician if he or she subjectively felt a sense of heartsink when seeing a patient on the schedule. No patient information was collected. For all visits involving these patients, clinicians in the intervention sites followed the BREATHE OUT procedure. They were instructed to complete the previsit 
Figure 1. Study protocol.

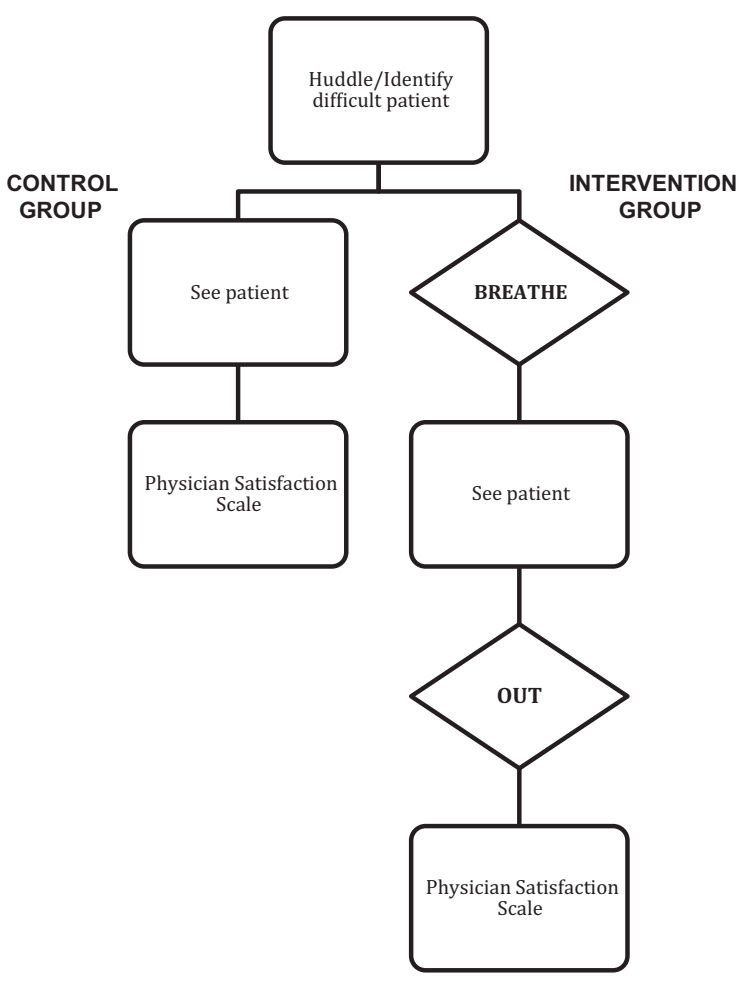

BREATHE mnemonic at the end of their huddle before seeing the patient and the postvisit OUT mnemonic immediately after the encounter. Clinicians at the control sites proceeded with these encounters as usual.

Following all these visits, all study participants completed the PSS. Clinicians could participate multiple times during the study period. Participation was voluntary. As a token of appreciation for participating in the study, the clinician and their back-office staff received chocolates. Weekly breakfasts also were provided to all 6 clinics to encourage study participation.

\section{Statistical Analysis}

We used $t$ tests (satisfaction scores) and $\chi^{2}$ tests (individual characteristics) to test for differences between intervention and control clinics. The analysis included 3-level hierarchical linear modeling (HLM) to model intervention effects at the visit level while incorporating the nesting of visits within clinician, clinicians within clinic, and clinics within the intervention and control groups. HLM is a widely used statistical method that allowed us to address the clustered data in this study, which are naturally nested (ie, individual, clinic, randomiza- tion group). Each physician-patient encounter $(\mathrm{n}=112)$ was coded with a physician indicator variable. Encounters were grouped by physician to create a second level of analysis, which included 57 unique physicians. The HLM statistical model calculated a variance term at the encounter, physician, and clinic levels. Control variables entered at the physician level included physician sex, job title, and years of experience. An indicator variable of intervention (1) or control physician (0) was entered at the physician level and evaluated for statistical significance to determine the primary outcome.

\section{Results}

Of 138 total eligible clinicians, 57 participated over the 5 -week period, resulting in a $41 \%$ overall participation rate. While each clinician saw at least 1 heartsink patient, some chose to participate more than once (a maximum of 6 times), resulting in a total of 112 observations. The intervention group's response rate was slightly higher (31 of 65 eligible clinicians [48\%] compared with $36 \%$ among the control group), although this was not statistically significant. Responders and nonresponders did not differ by sex or job title.

Overall, the 6 clinics had a mean of 18,400 annual visits, and the breakdown of insurance for patients was $38 \%$ health maintenance organization, $30 \%$ Medicare/Medicaid, 28\% fee-for service, and $5 \%$ other. There were no statistically significant differences between the experimental and control clinics on these variables. Table 1 provides characteristics of the study clinicians by intervention group status. Intervention and control clinics did not differ on observable clinic characteristics. Overall, $63 \%$ of the participating clinicians were women and half of the participants were resident physicians or fellows; $40 \%$ were attending physicians and $10 \%$ were nurse practitioners or physician assistants. There were no statistically significant differences in clinicians' characteristics between the intervention and control groups.

Table 2 shows the PSS score for the unadjusted intervention group reflected higher satisfaction compared with the control group (36.6 \pm 7.6 vs $42.8 \pm 8.6$ points [a lower PSS score indicates more positive/favorable outcomes]; $P<.001$ ). Female physicians demonstrated lower satisfaction on the PSS scale compared with their male counterparts (mean, $40.4 \pm 8.3$ vs $38.7 \pm 8.8$ points; $P=.03$ ). 
Table 1. Individual Characteristics of the BREATHE OUT Study Sample $(\mathrm{N}=57)$

\begin{tabular}{|c|c|c|c|c|}
\hline Variable & Total $(\mathrm{N}=57)$ & $\begin{array}{l}\text { Control Group } \\
\quad(\mathrm{n}=26)\end{array}$ & $\begin{array}{l}\text { Experimental Group } \\
\qquad(\mathrm{n}=31)\end{array}$ & $P$ Value \\
\hline Sex & & & & .19 \\
\hline Male & $21(36.8)$ & $12(46.1)$ & $9(29)$ & \\
\hline Female & $36(63.2)$ & $14(53.9)$ & $22(71)$ & \\
\hline Job title & & & & .52 \\
\hline Fellows/residents & $29(51)$ & $13(50)$ & $16(51.6)$ & \\
\hline Attendings & $22(38.5)$ & $11(42.3)$ & $11(35.4)$ & \\
\hline Other (eg, NP, PA) & $6(10.5)$ & $2(7.7)$ & $4(13)$ & \\
\hline Expertise level & & & & .57 \\
\hline \multicolumn{5}{|l|}{ Attendings } \\
\hline $0-5$ years' experience & $5(8.8)$ & $1(3.9)$ & $4(12.9)$ & \\
\hline 6-9 years' experience & $5(8.8)$ & $3(11.5)$ & $2(6.5)$ & \\
\hline$\geq 10$ years' experience & $12(21.1)$ & $7(26.9)$ & $5(16.1)$ & \\
\hline Other (eg, NP, PA) & $6(10.5)$ & $2(7.7)$ & $4(12.9)$ & \\
\hline \multicolumn{5}{|l|}{ Postgraduate year } \\
\hline 1 & $6(10.5)$ & $2(7.7)$ & $4(12.9)$ & \\
\hline 2 & $11(19.3)$ & $5(19.2)$ & $6(19.3)$ & \\
\hline 3 & $10(17.5)$ & $6(23.1)$ & $4(12.9)$ & \\
\hline Fellows & $2(3.5)$ & $0(0)$ & $2(6.5)$ & \\
\hline
\end{tabular}

Data are $\mathrm{n}(\%)$. Subjects in the control and experimental groups provided multiple observations for the study outcome measured with Physician Satisfaction Scale.

$\mathrm{NP}$, nurse practitioner; PA, physician assistant.

The total PSS scores did not differ by job title $(P=$ .12). PSS scores among intervention group participants remained improved compared with control

Table 2. Unadjusted Total Physician Satisfaction Scale Scores by Randomization Group, Sex, and Job Title $(\mathrm{N}=112)$

\begin{tabular}{lccc}
\hline & $\begin{array}{c}\text { Participants } \\
\text { (n) }\end{array}$ & $\begin{array}{c}\text { Total PSS } \\
\text { Score, } \\
\text { Mean (SD) }\end{array}$ & $\begin{array}{c}P \\
\text { Value }^{\dagger}\end{array}$ \\
\hline $\begin{array}{l}\text { Randomization group } \\
\quad \text { Intervention }\end{array}$ & & & \\
$\quad$ Control & 66 & $36.6(7.6)$ & $<.001$ \\
Sex & 46 & $42.8(8.6)$ & \\
$\quad$ Male & & & \\
$\quad$ Female & 37 & $38.7(8.8)$ & .03 \\
Job title & 75 & $40.4(8.3)$ & \\
$\quad$ Attendings & & & \\
$\quad$ Other (NP, PA) & 50 & $37.5(9.1)$ & .12 \\
$\quad$ Residents & 15 & $38.8(7.3)$ & \\
$\quad$ Fellows & 44 & $41.4(8.2)$ & \\
\hline
\end{tabular}

*A lower Physician Satisfaction Scale (PSS) score indicates more positive/favorable outcomes.

${ }^{+}$One-way analysis of variance.

${ }^{\ddagger}$ Subjects provided multiple PSS observations.

$\mathrm{NP}$, nurse practitioner; PA, physician assistant; SD, standard deviation. participants when the analysis was adjusted for sex, clinic effects, job title, and multiple observations in a 3-level HLM $(P=.02$; Table 3$)$.

Of note, the HLM analysis also showed that female respondents were significantly less satisfied than male clinicians following "difficult patient" visits $(P=.009)$. There were no significant differences in PSS scores based on job title.

Table 3. Hierarchical Linear Model Regression Coefficients for the Intervention's Effect on the Total Score of the Physician Satisfaction Scale in the BREATHE OUT Study After Adjusting for Sex, Experience, Clinic Effects, and Multiple Observations per Clinician $(\mathrm{N}=112)$

\begin{tabular}{lccc}
\hline Characteristics & $\begin{array}{c}\text { Regression } \\
\text { Coefficient }\end{array}$ & $\begin{array}{c}\text { Standard } \\
\text { Error }\end{array}$ & $\begin{array}{c}P \\
\text { Value }\end{array}$ \\
\hline Intercept & 42.66 & 1.26 & \\
Experimental & -5.86 & 1.66 & .02 \\
Fellow/resident & 3.99 & 3.21 & .22 \\
Attending & 0.82 & 3.12 & .79 \\
Other (NP, PA) & - & - & - \\
Female sex & 5.02 & 1.83 & .009 \\
Male sex & - & - & - \\
\hline
\end{tabular}

NP, nurse practitioner; PA, physician assistant. 


\section{Discussion}

The principal finding of this study is that the BREATHE OUT protocol increased clinician satisfaction with difficult patient encounters compared with routine practice not involving a reflective procedure. Because interactions with these complicated and difficult patients are highly prevalent in clinical practices ${ }^{1,2}$ and are associated with clinician burnout, ${ }^{8}$ BREATHE OUT may offer a strategy to counteract the compassion fatigue associated with difficult patients described by Elder et al. ${ }^{11}$

The BREATHE OUT intervention grounds itself in aspects of both a patient-centered psychosocial framework and physician-oriented self-reflective model. The latter tradition originates within a psychoanalytic framework created by Michael Balint a half century ago. Kroenke ${ }^{18}$ more recently reflected that ". . . difficulty is dyadic, a consequence of both patient and physician factors. Each party brought something to the table." As many as 16 physical symptoms are associated with patients being difficult and exacerbate differences between expectations of patients and clinicians, further corroborating the need to engage the patient in a patient-centered framework. ${ }^{5}$ Some components of the BREATHE OUT protocol, such as paying attention to breathing, are found in mindful practice that has been shown to enhance patient-centered communication and decrease clinician burnout. ${ }^{19,20}$ BREATHE OUT, however, does not require its user to have a background in meditative practice. We developed the BREATHE OUT intervention to focus on multiple domains both before and after a visit. It is possible that some domains may be more powerful in promoting physician satisfaction than others. We believe, however, that there is value in using the combined domains because they mirror the strategies used by "excellent" seasoned physicians ${ }^{9}$ who are known to work successfully with difficult patients. These include asking questions that reflect on bias, emotional content, breathing, and an openness to the unexpected.

BREATHE OUT was a helpful intervention for well-experienced faculty as well as clinical learners. Future studies may wish to introduce BREATHE OUT to other members of the health care community, such as front and back office clinic staff, to improve their satisfaction with their daily interactions with these challenging patients. Nurses, for example, often find themselves on the frontlines of care of difficult patients. One survey of intensive care unit, emergency department, and medical/surgical nurses found that $64 \%$ reported being physically assaulted and $88 \%$ verbally assaulted at least once during a 1-year period. ${ }^{21}$ An intervention that promotes pause and reflection may help deescalate such charged scenarios.

Although patients were not formally assessed in this study, there is evidence that patients who are considered difficult tend to be less satisfied with their clinicians than nondifficult patients ${ }^{1,7}$ and may benefit from a more circumspect approach to clinical care. In a pilot study exploring how difficult patients perceive the more relational aspects of the clinician-patient dyad, however, we found that difficult patients reported having an easier time communicating with their resident physician compared with nondifficult patients. ${ }^{22}$ While clinicians may be the more distressed party in these dyads, in future studies it would still be interesting to see whether using BREATHE OUT to promote physician self-reflection and find positive aspects of these encounters would lead to higher patient satisfaction.

Our study also demonstrated that female clinicians express greater dissatisfaction with their difficult patients than male clinicians. While the BREATHE OUT intervention helped female clinicians with their dissatisfaction, women continued to lag behind men in their PSS scores. Our results are consistent with those of other studies that showed that female clinicians tended to perceive themselves as having more difficult patients and experience greater burnout than male clinicians $(28 \%$ vs $21 \% ; P<.1) .{ }^{8,23}$ This is a preliminary finding because of the relatively small sample size and $P$ value when corrected for multiple comparisons $(P=.03)$. More research is needed to explore this gender discrepancy; over half of all primary care residents are women ${ }^{24}$ and thus are widely engaged in the care of difficult patients.

Study strengths include the randomized controlled design using a new procedure rooted in a patient-centered model of care, along with use of a validated outcome measure and a multivariate statistical analysis. We had a reasonable study participation rate of $>40 \%$, and these participants were representative of the clinic workforce with effective randomization. Since this study was conducted 
within a teaching setting, it provides a backdrop for a potential curricular innovation to assist clinician learners and their teachers with improving their relationships with heartsink patients.

Lack of inclusion of the 10-item Difficult Doctor Patient Relationship Questionnaire, ${ }^{25}$ which assesses the clinician-experienced sense of difficulty with patients, is a limitation of this study and needs to be addressed in future studies. Other limitations include a potential lack of geographic generalizability. Although rural, suburban, and urban sites were incorporated into our study design, all study sites were based in the Midwest and so may not apply more broadly. This study also was conducted in a residency setting so may not be generalizable to nonteaching practices. Because of limited racial and ethnic diversity among clinicians in the clinics studied, we could not explore the influence of race on study findings. In addition, we did not collect any objective data on the patients or patient visits, which may be considered for future studies. Finally, the PSS does not provide clinically significant cutoff points, which precluded us from examining changes in PSS scores in relation to patient outcomes.

The BREATHE OUT intervention increased clinician satisfaction with difficult patients, suggesting that its use could be part of an overall strategy toward a more positive perception of the patient and the encounter. This implies a trend toward perceiving the patient as less difficult and may lead to improved doctor-patient relationships and decreased clinician burnout.

\section{Conclusion}

This study demonstrated that clinician satisfaction with difficult patient encounters was significantly improved by applying the BREATHE OUT protocol. We hope that the study results encourage clinicians to use this intervention as a strategy toward developing greater success in working with difficult patients.

The authors thank Mary Beth Plane and Terry Little for their administrative support. The authors also thank Drs. John Frey, David Rabago, Mindy Smith, and Aleksandra Zgierska for their guidance and careful editing.

\section{References}

1. Hahn SR, Kroenke K, Spitzer RL, et al. The difficult patient: prevalence, psychopathology and functional impairment. J Gen Intern Med 1996;11:1-8.
2. Jackson JL, Kroenke K. Difficult patient encounters in the ambulatory clinic. Arch Intern Med 1999;159: 1069-75.

3. O'Dowd TC. Five years of heartsink patients in general practice. BMJ 1988;297:528-30.

4. Zuger A. The difficult patient, a problem old as history (or older). New York Times, March 6, 2007.

5. Hahn SR. Physical symptoms and physician-experienced difficulty in the physician patient relationship. Ann Intern Med 2001;134(9 Part 2):897-904.

6. Krebs EE, Garrett JM, Konrad TR. The difficult doctor? Characteristics of physicians who report frustration with patients: an analysis of survey data. BMC Health Serv Res 2006;6:128-35.

7. Hinchey SA, Jackson JL. A cohort study assessing difficult patient encounters in a walk-in primary care clinic, predictors and outcomes. J Gen Intern Med 2011;26:588-94.

8. An PG, Rabatin JS, Manwell LB, Linzer M, Brown RL, Schwartz MD. Burden of difficult encounters in primary care: data from the minimizing error, maximizing outcomes study. Arch Intern Med 2009;169: $410-14$.

9. Balint M. The doctor, his patient, and the illness. Lancet 1955;265:683-8.

10. Balint M. The doctor, his patient and the illness. London: Pitman Medical; 1957.

11. Elder N, Ricer R, Tobias B. How respected family physicians manage difficult patient encounters. J Am Board Fam Med 2006;19:533-41.

12. Stein HF. Reframing Balint: thoughts on family medicine departmental Balint groups. Fam Med 2003;35:289-90.

13. Shore BE, Franks P. Physician satisfaction with patient encounters: reliability and validity of an encounter-specific questionnaire. Med Care 1986;24: $580-9$.

14. Epstein RM. Mindful practice. JAMA 1999;282: 833-9.

15. Lewin S, Skea Z, Entwistle VA, Zwarenstein M, Dick J. Interventions for providers to promote a patient-centered approach in clinical consultations. Cochrane Database Syst Rev 2009;(4):CD003267.

16. Ashworth CD, Williamson P, Montano D. A scale to measure physician beliefs about psychosocial aspects of care. Soc Sci Med 1984;19:1235-8.

17. Levinson W, Roter DL, Mullooly JP, Dull VT, Frankel RM. Physician-patient communication: the relationship with malpractice claims among primary care physicians and surgeons. JAMA 1997;277: 553-9.

18. Kroenke K. Unburdening the difficult clinical encounter. Arch Intern Med 2009;169:333-4. 
19. Beach MC, Roter D, Korthuis PT, et al. A multicenter study of physician mindfulness and health care quality. Ann Fam Med 2013;11:421-8.

20. Fortney L, Luchterhand C, Zakletskaia L, Zgierska A, Rakel D. Abbreviated mindfulness intervention for job satisfaction, quality of life, and compassion in primary care clinicians: a pilot study. Ann Fam Med 2013;11:412-20.

21. May DD, Grubbs LM. The extent, nature, and precipitating factors of nurse assault among three groups of registered nurses in a regional medical center. J Emerg Nurs 2002;28:11-7.

22. Edgoose JY, Regner CJ, Zakletskaia LI. Difficult patients: exploring the patient perspective. Fam Med 2014;46:335-9.

23. Linzer M, McMurray JE, Visser MR, Oort FJ, Smets $\mathrm{E}$, deHaes HC. Sex differences in physician burnout in the United States and the Netherlands. J Am Med Womens Assoc 2002;57:191-3.

24. Brotherton SE, Rockey PH, Etzel SI. US graduate medical education, 2004-2005: trends in primary care specialties. JAMA 2005;294:1075-82.

25. Hahn SR, Thompson KS, Wills TA, Stern V, Budner NS. The difficult doctor-patient relationship: somatization, personality and psychopathology. J Clin Epidemiol 1994;47:647-57.

\section{Appendix}

BREATHE OUT

Instructions for Use: Identify a "difficult" patient during your huddle and complete the BREATHE previsit questionnaire before seeing the patient.
Immediately after the encounter, complete the OUT postvisit questionnaire.

\section{Previsit Questionnaire (BREATHE)}

1. List at least one Bias/assumption you have about this patient.

2. REflect upon why you identify this patient as "difficult."

3. List one thing you'd like to Accomplish today.

4. THink about one question you'd like to address today that would enable you to further explore your assumptions.

Please ensure you review some bumanizing aspect(s) of the social bistory with the patient that goes beyond a substance use screen. Consider recording this in EPIC's "Social Documentation" of the "History" section so that you can refer to this during future visits.

5. Stop before you Enter the patient room and take 3 deep breaths (in through your nose and out through your mouth).

\section{Postvisit Questionnaire (OUT)}

1. Reflect on the Outcome of the encounter.

a. From the patient's perspective: What was their agenda?

b. From your perspective: Did you accomplish your agenda? If not, how do you feel about it today?

2. Did you learn anything Unexpected?

3. List one thing you look forward to addressing if you were to run into this patient Tomorrow. 